\title{
On the Effective Path of Cultivating Research Tutors Under the Background of Study Travel
}

\author{
Yun Peng ${ }^{1}$ \\ ${ }^{1}$ Jiangxi Vocational Technical College of Industry \& Trade, Jiangxi,330103 \\ 739960103@qq.com

\begin{abstract}
With the continuous development of China's economy, all walks of life in China are blooming, and the demand for talents in the industry is constantly changing. As an evolving industry, research and learning travel has gradually attracted people's attention. This paper mainly studies the training plan of research and learning Tutors under the background of research and learning travel. As a kind of outdoor practical education activity combining the nature of research and tourism, the smooth development of research travel is of great significance. For research travel, research tutor occupies an important position and has an important influence on the formation of the whole research travel. Therefore, the cultivation of research tutors is very important. This paper also studies the talent cultivation strategy of research tutors based on this background.
\end{abstract}

Keywords: research travel, Research tutor, personnel training

\section{试论研学旅行背景下培养研学导师的有效路径}

彭芸 ${ }^{1}$

1 江西工业贸易职业技术学院江西南昌 330103

739960103@qq.com

摘 要:

随着我国经济的不断发展, 我国各行各业也百花齐放, 行业人才需求也不断的改变。研学旅行作为一个演变 的行业也逐渐受到大家的关注, 本文主要研究研学旅行背景下研学导师人才的培养计划。作为一种研究性质 与旅游性质相结合的户外实践教育活动, 研学旅行的顺利展开拥有着十分重要的意义, 对于研学旅行而言, 研学导师占据着重要地位, 他对整个研学旅行的形成有着重要的影响。因此, 研学导师的培养是十分重要的, 本文也基于此背景下进行研学导师的人才培养策略研究。

关键词: 研学旅行; 研学导师; 人才培养

\section{1. 研学旅行的内涵、特征及意义}

现代旅游与现代教育相结合, 产生了新的旅游 模式, 研学旅行便是其中之一, 作为新的旅游模式, 研学旅游与教育相结合, 旨在通过旅游当中的见闻 进行有规律的学习, 使得学生的学习更具有组织性 以及体验性, 给学生带来更好的学习氛围。同时, 也有助于学生创造能力的形成与培养。

\section{1. 研学旅行的内涵}

研学旅行有两方面的内涵, 它分为广义内涵和 狭义内涵, 从广义内涵来看, 研学旅行主要是指旅 游者在任意时间段离开自己的居住地, 前往其他地 方进行文化活动, 并从当中获得一定的知识感悟, 没有一定的组织形式，也没有一定的人群限制。而 狭义的研学旅行, 主要是指学生通过学校或者教育 部组织的以旅游形式为主要形式的课外实践活动, 来获取一定知识的行为。这种旅游方式能够促进学 生与大自然亲近, 同时能够培养学生的团队意识, 
并且使学生的身心健康得到一定的发展。除此之外, 还能够开拓学生的思维, 使学生更具有创造性。本 文也主要是以狭义的研学旅行作为研究对象进行详 细的探究。

\section{2. 研学旅行的特征}

\subsection{1. 研学旅行具有体验性}

研学旅行是通过旅游的手段, 让学生与自然界 与社会进行有效的接触, 使得学生能够在自然界中 以及社会中学到书本上学习不到的知识, 这样有利 于学生思维的开拓以提高。这种教学方法与教学模 式是更具有体验性的, 它使得学生能够切身的到当 时所处的环境当中去进行学习和感悟, 而非在一个 想象空间当中进行领悟。

\subsection{2. 研学旅行具有课程性}

研学旅行还具有课程性, 这是由于研学旅行是 由学校或者相关的教育部门组织的, 同时, 要求学 生严格按照一定的旅游计划进行旅游, 在旅游的过 程中要得到此次旅游的感悟, 而并非仅仅只局限于 玩乐。

\subsection{3. 研学旅行具有教育性}

研学旅行是具有教育意义的, 对于学生而言, 通过研学旅行, 可以让自己与大自然与社会进行进 一步的接触, 使得自己能够在大自然和社会中学习 一定的知识。同时, 研学导师在整个研学旅行的过 程中, 也会结合旅行地点的实际情况以及学生的个 人发展情况, 有目的的进行知识教育。

\section{3. 研学旅行的意义}

在物质发展的今天，学生可以得到的资源也是 越来越多的, 但是学生的学习压力也是越来越大的, 学生如果仅仅只在课本上学习知识, 而不去关注外 界世界的变化, 不去从自然界中, 从社会当中给予 力量, 就会被这个时代所淘汰。同时, 只仅仅埋头 苦读, 局限于课本知识, 也会使得学生的身心健康 受到一定的损害。因此, 定期的进行研学旅行是有 其存在的必要性的。研学旅行通过旅行模式, 让学 生在自然界中、在社会中获取一定的知识, 汲取一 定的养分。这有利于学生身心健康的提高, 也有利 于学生眼界的开阔, 从而达到学习效果倍增的目的。 而且通过带领学生走进真正的名胜古迹, 真正的博 物馆, 能够让学生感受到真正文化的气息, 感受到 中华文化当中的那一份深厚后, 这样能为学生日后 的学习打下一定的基础, 也有利于学生学习兴趣的 培养, 对学生个人的发展是十分有益的。同时, 通 过旅行的模式, 还给学生提供了一个交流互助的平 台, 使得学生之间的情谊可以更加的深厚, 也让他
们能够在旅行当中意识到集体的作用以及集体合作 的作用。

\section{2. 研学导师核心素质分析}

武汉市研学旅行导师评定与服务规范中指出研 学导师是在研学旅行中, 具体制定或实施研学旅行 教育方案, 指导学生开展各类活动的专业人员。其 中, 了解服务机构研学导师及基、营地研学导师应 具备的条件, 和人才培养中对学生在旅行社和景区 就业的定位相结合, 对于设定研学导师方向具有很 强的指导意义。在研学旅行的整个环节中, 除了课 程的开发, 优秀的研学旅行导师是保证研学旅行质 量的重要因素。

\section{1. 熟知教育理念}

作为一名合格的研学旅行的导师, 他需要拥有 足够强的知识传递能力, 具有一定的教育理念, 能 够对学生进行教育, 同时能够拥有课程组织能力以 及课后的总结能力。对于研学旅行的执行者而言, 他们需要通过高质量的研学旅行活动培养学生的产 能力以及创造能力, 让学生能够在整个言辞旅行的 活动中真正受益。因此, 对于研学导师而言, 他需 要熟知教育理念, 同时能够很好的将教育理念传递 给学生。

\section{2. 具备安全意识}

其次, 作为研学旅行的主要项目负责人, 研学 旅行的老师应该具备强烈的安全意识。由于研学旅 行的课堂是一个开放性质的课堂, 而课堂当中的大 多数群众是中小学生, 因此, 研学导师必须具备强 烈的安全意识。只要这样才能够在面对安全问题以 及安全事件的过程中, 拥有一个快速的处理问题的 能力。除此之外, 研学导师还应当对旅途当中可能 发生的其他安全隐患以及卫生, 食品安全等负责。 研学导师在旅途当中还应当注意当地天气和交通以 及疾病等等, 以保证孩子们的身体安全。

\section{3. 注重沟通交流}

研学老师还需具备强烈的沟通交流能力。对于 研学导师而言, 在整个旅途当中, 他都得负责学生 的身心健康, 需要照顾学生的情绪, 同时还要防止 出现争吵等事件的发生。因此, 这就需要研学导师 拥有较强的交流沟通能力。能够将这个交流沟通能 力运用到研学旅行的过程中, 使得不同年龄阶段、 不同性别以及不同情绪的孩子们能够更好的在团队 中进行交流学习, 能够更好的融合于团队当中, 同 时也能够更好的通过与老师的对话进行学习和创造。 除此之外, 老师还要善于诱导孩子进行学习, 调动 孩子学习的热情和学习的积极性, 这样才能够让学 生从研学旅行当中获取更多的知识。 


\section{4. 提升讲解能力}

研学导师作为项目的主导者, 他应当承担学生 的学习任务。因此, 研学导师需要拥有较强的讲解 能力, 能够对是课外的事物以及生活当中所发生的 事件进行一定的讲解和输出, 让学生从大自然中, 从社会当中学习到书本上学习不到的知识。这是进 行研学旅行的主要目的, 因此提升研学导师的讲解 能力是十分有必要的。

\section{3. 当前研学旅行实施中存在的问题}

\section{1. 课程设计不完善}

研学课程设计不够完善, 大多数研学课程设计 往往过于形式化, 很少有真正的研学旅行, 能够做 到内容输出、教育理念传达和实现教学目标三者的 融合。并且在融合的过程中, 很少研学旅行能够对 孩子品格进行一定的培养，使得孩子的身心得到更 好的发展。因此, 学旅行课程设计应当进行一定的 改良, 使得研学旅行的课程更符合于研学的实际情 况, 让学生能够从真正的旅行当中学习到知识与及 经验。

\section{2. 安全保障体系不健全}

由于研学旅行是以旅行的形式, 让学生通过集 体旅行以及集体食宿的方式参加一系列的校外活动, 从而让学生学习到一定的课本外知识。而通过集体 旅行的方式需要拥有较强的安全保障体系, 但是对 于现阶段的研学旅行而言, 由于它的起步较晚, 他 的安全保障体系还是不够健全的。并且现阶段而言, 大多数学生都是独生子女, 因此很多家长对研学旅 行这一形式并不表示支持。并且由于春游安全隐患 事件的频繁发生, 这也使得研学旅行的口碑受到一 定的影响, 也导致了研学旅行的市场需求缩小, 而 市场需求缩小, 研学旅行进行改造的机会做变少, 安全体系更难以健全。

\section{3. 导师讲解水平不高}

老师的讲解水平也不够高, 由于研学导师在研 学旅行中占据着非常重要的地位, 它是使得学生获 得知识的主要桥梁。因此, 研学导师应该拥有较强 的讲解水平。然而在现阶段的市场下, 由于研学旅 行市场的需求较少, 且研学旅行市场还属于一个刚 开放的市场, 所以研学导师的水平普遍不是很高。 因此, 提高研学讲师的讲解水平是十分重要的, 这 样才能够让学生从旅行过程中的所见所闻中获取一 定的知识。

\section{4 研学旅行背景下的研学导师方向人才培养策 略}

\section{1. 保证高校稳定的双师队伍}

在进行研学导师方向人才的培养过程中, 应该 保证高效稳定的双师队伍。由于研学导师需要的能 力是足够强的实践组织能力和足够强的讲解能力, 就使得研学导师的培养不仅仅局限于课本, 还应当 在实践当中进行培养。因此, 在研学导师方向人才 培养的过程中, 应该建立双师型的教师队伍, 使得 研学导师人才能够在课本上学习一定的基础素质知 识, 培养专业的知识素养, 同时还应当在实践当中 学习更多的实践知识, 使得研学导师能够将自己所 学到的知识顺利地运用到实践当中去, 能够尽快地 组织学生进行研学活动, 并且能够使得整个研学活 动顺利的进行下去。

\section{2. 创建模块学习式的一体化课程体系}

在进行研学导师方向人才培养的过程中, 应当 建立模块学习是一体化的课程体系。对于研学导师 而言, 有需要的综合素质是十分高的。因为他不仅 要进行知识的传授, 还要保证学生的安全, 同时还 要进行学生的组织工作。因此, 在研学导师的培养 过程中应该实现教学模块一体化的课程培养模式。 同时在多方面都进行一定的培养, 实现研学导师全 面的培养。并且, 全方位的课程体系也要通过实践 的方式进行输出, 让研学导师人才在实践当中将所 学到的知识进行一定的融合, 并且需要对实践的方 式对融合后的实践作品进行打分, 这样才能够更加 有利于研学导师的全面发展和整体培养。

\section{3. 加大校企合作力度, 校企共建核心专业 课程}

在研学导师的培养过程中，应当加大校企合作 力度, 校企共建核心专业课程在进行课程。加大校 企合作力度, 主要是以下两个方面, 第一个方面是 高校与企业的合作力度，第二个方面是中小学于企 业的合作力度。在高校方面, 通过与企业进行合作, 定期的将学生送往企业进行一定的学习培养, 并且 通过企业老师定期的打分能够让真以及学校更清楚 的明白学生目前的学习情况, 能够对学生进一步的 进行培养。除此之外, 中小学也可以加大于企业的 合作, 定期的组织学生进行学研旅行, 当然这个组 织是以自愿为原则的。

在加大校企合作的过程中，可以使得高效的学 研人才进行一定的实践。同时，对于中小学生而言， 他们也有有更多形式的课程学习。因此, 在研学旅 行人才的培养过程中, 还可以加大校企合作的力度, 共建校企核心专业课程。 
在专业课程的构建方面，通过两校一起的不断 磨合, 可以明确整个研学旅行过程中, 最适合学生 的学习方案, 从而解决研学旅行课程不适合学生的 问题, 满足了对研学人才的培养计划。

\section{5. 结论:}

研学旅行作为一个新的市场需求, 它是符合时 代发展的, 并且研学旅行对于现代的学生教育而言 有着积极的作用。本文也基于此, 研究了研学旅行 的内涵, 特征以及研学旅行的意义, 并且分析了成 为研学导师需要的核心素质以及当前研学旅行过程 中存在的一系列问题, 并且针对这些问题给出了相 关的解决方法, 希望能够为研学旅行培养出优秀的 研学旅行导师人才, 从而使得学生能够通过研学旅 行的方式收获到课本上学不到的知识, 丰富自身的 人生阅历。

\section{REFERENCES}

[1] Qu Xiaoyi. Strategies for travel agencies to cope with opportunities and challenges from the perspective of research study travel -- a comparative study of research study travel and traditional tour guides based on policy analysis [J]. Journal of Lanzhou Institute of education, 2019,35 (12): 81-82

[2] $\mathrm{Hu}$ Chi. Research on the satisfaction degree of professional ability of research travel tutors based on SPSS -- Taking Wuhan as an example [J]. Journal of Wuhan Polytechnic, 2020,19 (5): 59-63

[3] Qu Xiaoyi, di Lei, Meng Yanhong. Exploration on the establishment of research tutor evaluation system from the perspective of research travel -- Based on the comparative study of research tutor and traditional tour guide $[\mathrm{J}]$. Science and education guide, 2020 (32): 55-56

[4] Zhang Lei. Practice and Thinking on the training mode of tour guide talents under the background of introducing enterprises to Campus -- Taking Jiangxi tourism and Business Vocational College as an example [J]. Journal of Baotou vocational and technical college, 2020,21 (3): 65-67

[5] Wang Xin, Ji Keling. Exploration and practice of the professional construction mechanism of "introducing enterprises into education, introducing enterprises into schools, and introducing banks into schools" -- Taking Automobile Engineering College of Jinan Technician College as an example [J]. Occupation, 2019,0 (30): 90-91

[6] Li guangkao, Gu Xiaoyi, Zheng Shaofeng. Research on the working mechanism of "three complete education" based on the tutorial group -- Taking Liming Vocational University as an example [J].
Journal of Huainan vocational and technical college, 2020,20 (1): 111-113

[7] Peng Lei, Chen Yan, Xu Weilong, Li Xing, Wang Jun. feasibility study on primary school students' study travel in Handan from the perspective of stakeholders: a case study of Zhuhe Road Primary School in Handan [J]. Journal of Handan University, 2020,30 (3): 116-120

[8] Xiang Chunyan, Zhou Chunyan. Development of research travel products based on red tourism resources: a case study of Chongqing Hongyan scenic spot [J]. Journal of Chongqing University of Arts and Sciences: Social Science Edition, 2021,40 (1): $68-79$

[9] Lin Jieling. One belt, one road, the development strategy of research and tourism market: a case study of Quanzhou, Fujian Province, [J]. Journal of Shanxi Cadre College of Economic Management, 2020,28 (4): 25-28.

[10] Chen Junling. On the construction of school research education system: a case study of "unity of knowledge and practice" in the third Experimental Primary School of Tong'an District, Xiamen [J]. Information technology education in primary and secondary schools, 2021 (2): 154-156

[11] Wan Tianhu, Li Chunxiang, Wang Luqing. Research on the design and implementation mode of research travel products in National Forest Park -- a case study of Yunbifeng in Shangrao, Jiangxi Province [J]. Western economic management forum, 2021,32 (2): 63-71

[12] Guan Changchun. Research on the value of Ideological and political education and the realization path of red research and study travel -Taking Sanming City as an example [J]. Journal of Taiyuan City Polytechnic, 2020,0 (1): 133-136 